\title{
A stochastic modeling framework for the Invitational Drought Tournament
}

\author{
G. Strickert ${ }^{\text {a }}$, A. Nazemi ${ }^{\text {b }}$ and L.E. Bradford ${ }^{c}$ \\ ${ }^{a}$ Global Institute for Water Security (GIWS), The School of Environment and Sustainability, The University \\ of Saskatchewan, Saskatoon Saskatchewan \\ ${ }^{b}$ Department of Building, Civil and Environmental Engineering, Concordia University, Montreal, Quebec \\ ${ }^{c}$ School of Public Health, University of Saskatchewan, Saskatoon, Saskatchewan
}

Email: graham.strickert@usask.ca

\begin{abstract}
Droughts are better defined as socio-hydrologic phenomena. On one hand, hydroclimatic extremes dictate the frequency and severity of droughts. On the other hand, management decisions alter associated impacts of droughts on coupled human-natural systems. Although hydrological aspects of drought have been explored in the past, the social dimension of drought management has received less attention. Drought management exhibits a complicated decision making process and is normally performed through a pluralistic decision making context, which involves a wide range of social groups with potential for competing interests. In order to explore and understand social relations within drought management, theoretical and modelling frameworks are required. Such a framework should be able to describe and model the human dimension of drought management. We argue that Cultural Theory, an existing conceptual framework to explain societal conflict over risk, can frame the social aspects within drought management practice. We introduce a dataset from an Invitational Drought Tournament event and demonstrate how Cultural Theory and stochastic modelling of participant's preferences explains the social relations empirically. We find that competition is an important social behavior within a drought management. The Invitational Drought Tournament captures important social relations that result in new and innovative management strategies to tackle drought risks by reducing probability/frequency and impact severity. The strategies put forward to manage the drought depend on the stage of drought, participants' insights and different ways of organizing. We show empirically that an autonomous regulatory framework is required to guide individual and institutional conflicts while allowing for pluralism in drought management practices.
\end{abstract}

Key points:

- We suggest more effort is needed to model the human dimensions of drought

- Cultural Theory is proposed as a useful lens to support analysis

- The Invitational Drought Tournament is outlined as a behavioural observatory

- Choices of participants in the game are used to create Empirical Cumulative Distribution Functions (ECDFs)

- ECDFs are used to simulate the game to fit the referee's scores

Keywords: Socio-hydrology, drought management practice, pluralistic decision making, cultural theory, Invitational Drought Tournament 


\section{INTRODUCTION}

Droughts occur as the result of extremely dry hydroclimatic conditions and substantially reduced water availability for societal and environmental productivity (Bonsal et al., 2011). As water underpins the most basic needs for human societies, droughts are associated with substantial vulnerabilities to human livelihoods. In fact, severe droughts led to some of the most catastrophic socio-economic disasters throughout history, such as the Northern Chinese Famine of 1876-79 (Hao et al., 2010) and North American Dust Bowl of 1930s (Schubert et al., 2004) both due to lack of water. More recently, from 1999 - 2004 a drought occurred in the Prairie Provinces costing $\$ 6$ billion (CAD) (Wheaton et al., 2008). Presently, a drought has resulted in the first ever water restrictions in the Californian agricultural sector (Nagourney, 2015). From the physical perspective droughts are natural phenomena, but in the social sphere, the drought management responses change the associated impacts on humans and the environment. Climate and hydrological sciences have resulted in an improved description (Wilhite and Glanz, 1985), quantification (Giacomelli, et al., 2008) and prediction of the impacts of drought on the water resources system (Nazemi et al., 2013), however, current and future drought impacts cannot be fully understood unless the human systems are taken into account (Gober and Wheater, 2014). In fact, due to the interconnection between human and water systems, it has been suggested that droughts are better explained as socio-hydrologic events rather than purely hydroclimatic extremes (Sivapalan et al., 2012). Interactions between natural and human systems have been also explored to some extent. For instance, Nazemi and Wheater (2015) demonstrate how human management can perturb terrestrial water cycles across various scales.

Studying the social aspects of drought requires a theoretical framework allowing the social aspects to be collected, and analyzed empirically. We argue that Cultural Theory, a conceptual framework to study selective attention to risks can describe the some of the social aspects of drought. This paper can be considered as a proof of concept based on the data from an Invitational Drought Tournament (see Hill et al., 2014). In Section 2, we briefly introduce Cultural Theory. In Section 3, we introduce our data with which we try to explore how Cultural Theory can describe the social interactions in a drought management workshop. In Section 4, the data are explored to identify the key social response to droughts. Section 5 discusses our results and shows why regulation is required to handle the individual and institutional competitions in the human dimensions of drought. Finally, Section 5 concludes our study and provides further remarks.

\subsection{CULTURAL THEORY}

Risk can be defined as the combination of the probability of an event and its negative consequences (UNISDR, 2009). During extreme and uncertain events, human views risk through perceptual filters (Adams, 1995; Adams and Thompson, 2002; Thompson, 2008ab). The filters are influenced by many parameters such as knowledge, psychology, economics and/or ideology; however, culture is the most inclusive aspect (Mars, 2008). What distinguishes culture from other aspects is the emphasis on social interactions. Structures of social organization give individuals and groups perceptions that reinforce structures in competition against alternative ones - people disagree in predictable ways (Verweij et al., 2011). Cultural Theory holds that there are five ways of organizing social life: individualism, hierarchy, egalitarian, fatalism, and autonomy/insight (Schwarz and Thompson, 1990; Adams, 1995). Individualism is low on both integration and regulation; while hierarchy combines high integration and regulation; egalitarian is high on integration but low on regulation; fatalism is high in regulation but low in integration; and insight/autonomy is neutral in both dimensions and observes the interactions between disparate ways of organizing (Thompson et al., 1990) (Figure 1).

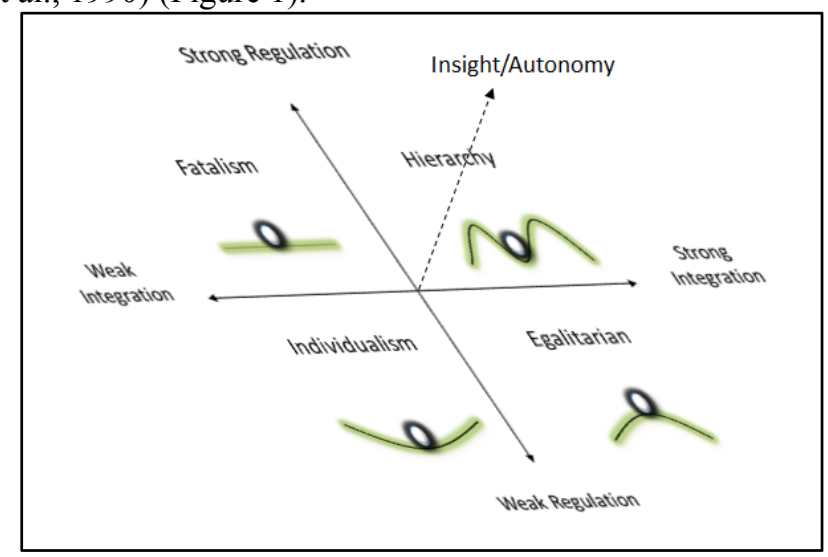

Figure 1. The five different ways of organizing according to Cultural Theory. 
"Each way of life undermines itself. Individualism would mean chaos without hierarchical authority to enforce contracts and repel enemies. To get work done and settle disputes the egalitarian order needs hierarchy, too. Hierarchies, in turn, would be stagnant without the creative energy of individualism, uncohesive without the binding force of equality, unstable without the passivity and acquiescence of fatalism. Dominant and subordinate ways of life thus exist in alliance yet this relationship is fragile, constantly shifting, constantly generating a societal environment conducive to change" (Schwartz, 1990, p. 765).

\section{THE INVITATIONAL DROUGHT TOURNAMENT}

The Invitational Drought Tournament (IDT) is a one-day workshop designed to enhance discussion among interdisciplinary groups about how to manage droughts (Hill et al., 2014). The Saskatoon Invitational Drought Tournament took place in March 2012. The tournament brought together teams of participants who were early career researchers (e.g. graduate students and postdoctoral fellows) from diverse backgrounds of study such as sustainable development, water resource management, agriculture, hydrology, geography and engineering. Teams were from four universities in the Canadian Prairie provinces, and were competing for small prizes and the title of "IDT 2012 Champions". The teams were from the University of Alberta ( 2 teams; A1, A2), University of Saskatchewan (1 team; S), University of Regina (1 team; R) and University of Manitoba (1 team; M). There were also four referees from western Canada and the USA who evaluated the performance of the teams. They included: one water engineer, two water scientists, and one agricultural specialist; all of the referees had working knowledge of drought mitigation and response. One month before the tournament, an information package was sent to familiarize the participants with the context and background, the process of the IDT, and pre-defined adaptation strategies that teams could use (see Strickert et al., 2013; Hill et al., 2014).

\subsection{The Saskatoon IDT}

The tournament was played in three iterative rounds. At the beginning of each round teams are given a drought scenario for the "Oxbow' watershed, which was based on the Saskatchewan River Basin, in Canada. Each scenario included information about streamflow, snowpack, precipitation, temperature and water demand, as well as social, economic and environmental impacts of the drought. At the start of the tournament teams chose from fifteen pre-defined adaptation strategies outlined in the information package. Each strategy differed by adaptation category (or process), type (in terms of temporality), and actual on-the-ground actions. The strategies broadly included infrastructure projects and mechanized manners of influencing water courses, economic relief efforts, research and development options, and consultation choices. The costs and limited budgets meant that teams had to be selective about the strategies they chose each round. Teams were also allowed to put forward novel adaptation strategies (i.e. innovations) but had to discuss these with the referees and the referees estimated the costs of these options. At the end of each round teams submitted their suite of adaptation strategies to the referees and presented their justifications for their choices to the other teams. The referees, individuals and teams evaluated the choices and justifications using a participatory scoring system that gauges the ability of the management plan to reduce social, environmental and economic costs associated with the drought. There are three parts to the scoring:

1. Individuals gave each team including their own a score from 1-9 (where 1 is the most effective suite of strategies for reducing risk, and 9 is the least effective for reducing risk).

2. Teams deliberated and then provided one team score for each team using the same scale.

3. Referees give each team a score using the same scale.

The scores were weighted in the following way, Individuals $25 \%$, Teams $25 \%$, and Referees $50 \%$. Individuals and teams were given an equal vote to balance-out group processes, allowed for the examination of individual versus group preferences. Referees were given the balance of power because they were considered independent experts in drought mitigation and response. The team with the lowest score (i.e. lowest risk to the consequences of the drought) at the end of the game wins.

\subsection{Team Choices}

All of the teams chose multiple adaptation strategies in each round (Table 1). There are some interesting features in the selection of adaptation strategies. First, all the teams selected some of the adaptations, while other adaptations were selected by none of the teams. Recordings of group discussions found that some options were seen as cultural plausible or implausible. For instance, all teams advised rationing water during rounds 2 and 3, while diverting water from another basin was not used by any of the teams during the game. 
Another feature is the gradual shift from selecting more long-term strategies to more short-term ones, possibly due to the competitive nature of the tournament.

Table 1. Adaptation Options and Teams Choices in Each Round.

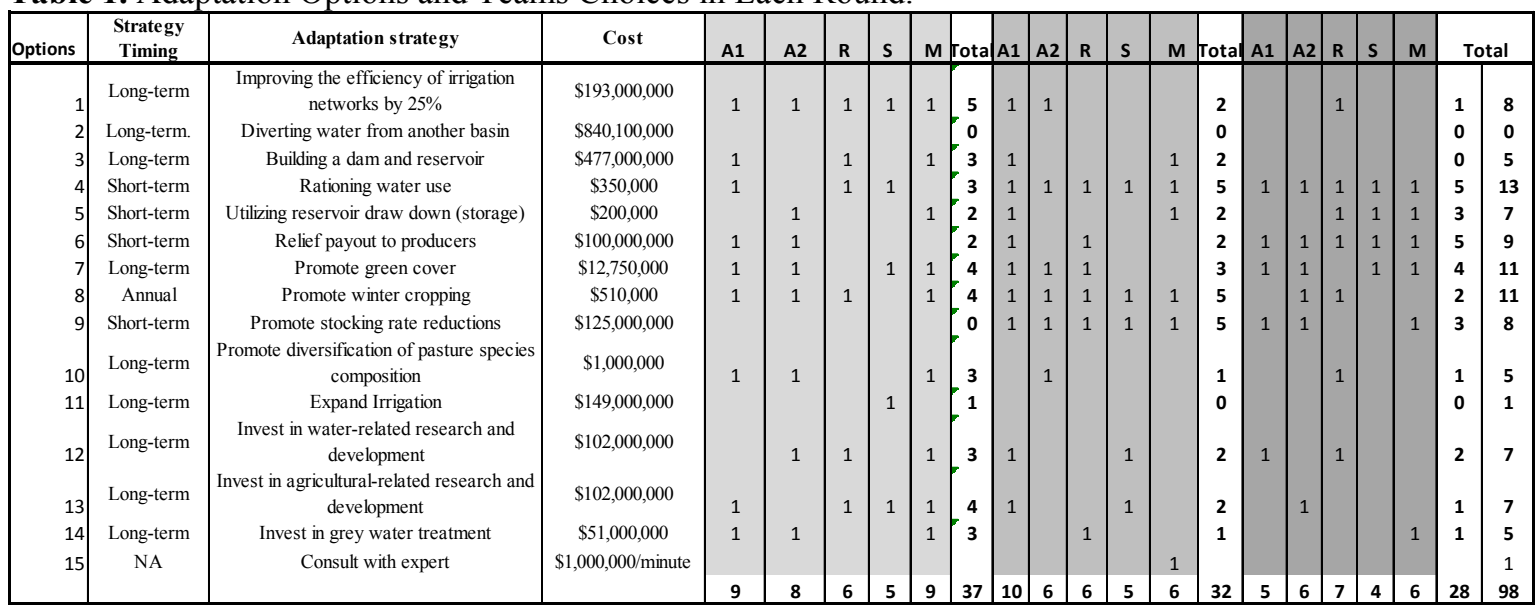

\subsection{Social Competition}

In the tournament, a competitive atmosphere emerged. The dynamics of decision making shifted from choosing the best adaptation strategies towards preserving the team score. To investigate the differences between group and individuals' votes in the IDT, the frequency of scores given by individuals, teams and the referees were examined to look for patterns (Figure 2). Four distinct scoring patterns were identified including (2a) individual and teams provided good scores for their own team; (2b) individuals and teams provided worse scores for the 'winner-so-far'; (2c) individuals and teams provided fairer scores for nonwinning teams and (4) referees scored more equitably (Figure 2). Both individuals and teams gave their own teams higher scores in all rounds with a few 'conscience' votes, and a few initial confusion votes (voting 9 as a high score). In contrast, individuals and teams gave the winner after each round a poorer score. The winner after each round was penalized more by teams than individuals. Individuals and teams gave the "other" team (lowest ranked) a fairer distribution of scores, nurturing the losing teams. The referee's scores were distributed more evenly and with less variation in the votes and with a high concentration around the central value.
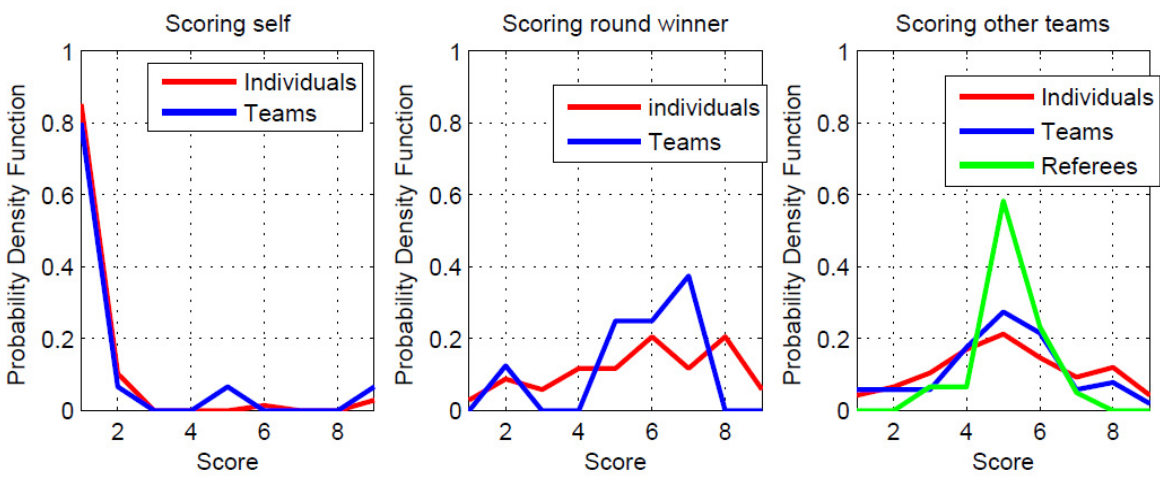

Figure 2. Probability density function for scoring self, round winner and other teams.

\section{TOWARDS AN IMPROVED PLURALIST APPROACH TO DROUGHT}

The scoring patterns emphasize the differences between how teams and individuals evaluate other teams' strategies. Accordingly, it is necessary to quantify how these differences can change the outcome of collaborative decision making. The self-centered votes of teams and individuals may hinder the selection of the best suite of adaptation strategies; the central goal of the tournament. To limit the influence of selfserving behaviour, a robust regulatory system, in the form of weighted scoring is therefore needed to retain the competition while also enabling the identification of the best suite of adaptations.

In order to identify the differences between individual, group, and referee scores, a stochastic sensitivity analysis was run to identify how different weighting schemes change the outcome of the tournament. The extracted scoring patterns (Figure 2 above) were treated as Empirical Cumulative Distribution Functions 
(ECDFs); then used as a basis for generating random sequences of scores in a Monte Carlo sampling scheme (Metropolis and Ulam, 1949) to reconstruct the IDT. Random scores are aggregated through the weighting system to identify the winner of the game. By considering different weighting for individuals and teams, we monitored how the final decision can diverge from the referees' choice, given the assumption that the referees in the game represent a less-biased (i.e. not self-interested) evaluator of adaptation strategies.

\subsection{Stochastic Simulations of the Votes}

The sampling scheme tests if the winner is the same as the referee's choice of the winner after each round. Figure 3 shows sixteen weighting systems for aggregating the scores. For each weighting system and each round, the scores from teams, individuals and referees were randomly generated using the inverse ECDFs associated with the four scoring patterns explained in Figure 3. Five teams were used for stochastic reconstruction; therefore, the lowest expected probability for the weighting system to identify the same winner as the referees' is 0.2 . A system with probability of 0.5 or more can approximate the referee's preference. One-hundred simulations of the IDT were used to compute the probability of tracing the referees' preference for each weighting system. The simulations for each weighting system were repeated 100 times to extract the uncertainties in the computed probabilities. There are some interesting observations in Figure 3. First, in the case of weighting systems W12 to W16 tracing the referees' preference is likely a matter of random chance. Some regulation (i.e., hierarchy) is needed to regulate the competitiveness and conflicting interests among teams. Second, it seems that the competitiveness at the teams' level is more than what it is at the individuals'. For instance, for W10 and W11 both have the referees' weights set at 0.25 but, differ by flipping the weights of 0.25 and 0.5 for individuals' and teams' votes. Based on the simulation results, the chance of tracing the referee's preference in these circumstances is significantly higher when the individuals' perspective is weightier. This is perhaps due to the fact that at the teams' level the main task seems to be winning the game rather than preserving the best drought management practices proposed by other teams. The same conclusion can be made for W2 vs. W3, W4 vs. W5 and W8 vs. W9. Among the simulated weighting systems, W6 seems to be the most appropriate way to aggregate the votes as it (i) takes into account all the voices and (ii) most closely matches the referee scores between 0.58 to 0.85 probabilities with a mean value of 0.7 . The use of an evenly split weighting between individuals, team and referees (W7), will trace the insight of the referees (mean probability of 0.55 ), but in approximately $30 \%$ of the realizations the chance is less than 0.5 . The expected values go below the 0.5 probability threshold for W8 to W16, which shows that the competitiveness among the teams and individuals become the dominant feature of the decision making process.

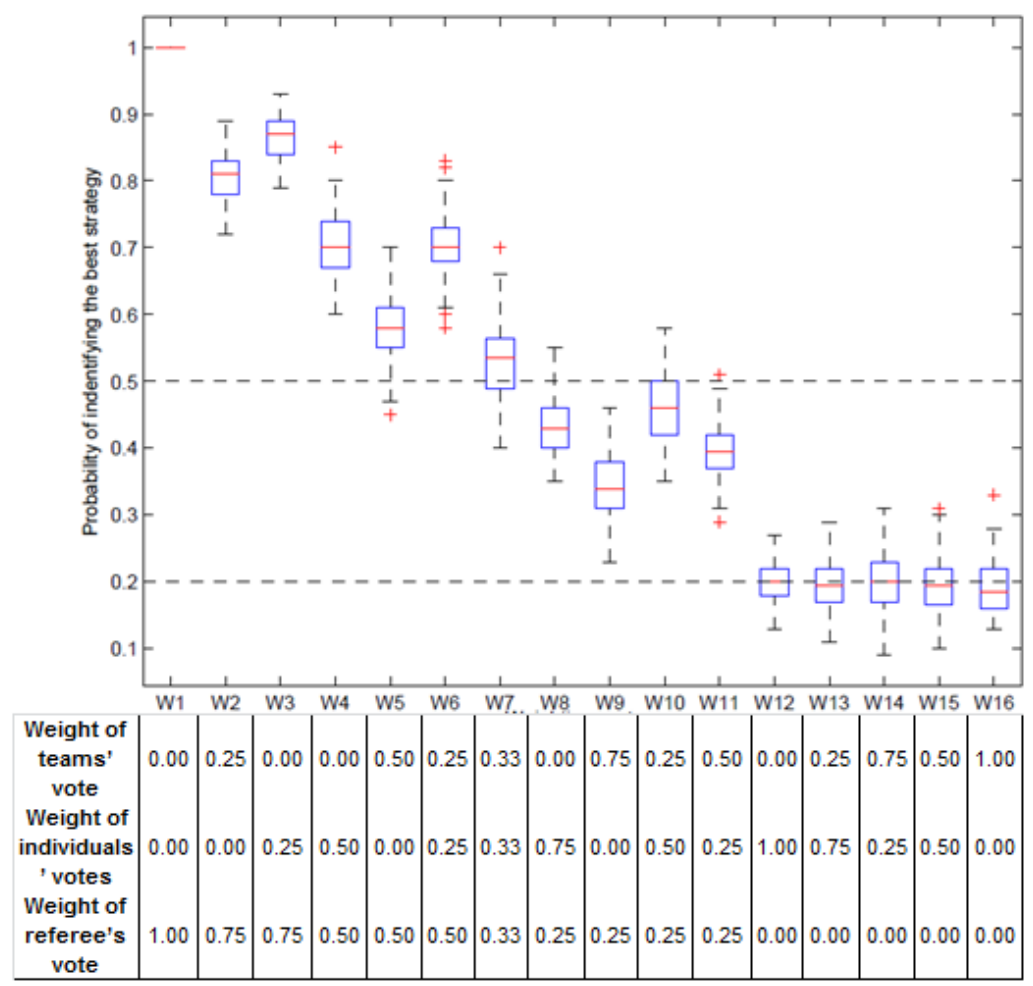

Figure 3. The probability for the winner of the game to be the same as the referee's preference. 


\section{DISCUSSION AND CONCLUSIONS}

Observing the social dynamics of decision making during scenario simulations provides important lessons for improving actual water resource management during times of stress. By analyzing the 2012 IDT in Saskatoon, we explored the different scores between individuals and teams. Cultural Theory's ways of organizing help us to understand three social dimensions within the tournament: 1) the selection of adaptation options, 2) the individual and teams scoring patterns, and 3) the simulations based on the teams scoring patterns. First, the strategy of diverting water from another basin was rejected by all teams. The team discussions felt it was a politically infeasible strategy; it assumes nature as robust, while also imposes topdown unilateral water withdrawal respectively, while rejecting the egalitarian perspective. In addition, as the drought persisted and resources became scarce, the teams shifted from long-term strategies, more commonly associated with hierarchy and egalitarians ways of organizing toward more short-term strategies, namely, individualism and fatalist strategies. Second, the scoring patterns also shed light on the social interactions in the tournament. There was a high level of self-promotion (individualism) and regulation (hierarchy) as the result of the competition among participants. To counter these patterns, an autonomous regulation scheme was developed to see what level of weighting is most likely to identify the best strategy, assuming that the referees are autonomous/independent actors. Although there is overlap between the simulated votes, the individuals stand a better chance of selecting the best adaptation strategy, perhaps by drawing on pluralistic approaches to adaptation. This research is of value for those undergoing or planning for drought decision making; it reminds us that individuals, groups and 'experts' may act differently in the context of competition for vital resources. Often decisions are made by groups of teams of people without considering the value of independent views. Encouraging all voices to contribute to decision-making is essential. Individuals and groups shift focus as competitions deepen, fairness among players wanes, and strategies focusing on curbing immediate needs for the drought, and for self- and group-preservation become dominant. The tournament's round-by-round scoring also meant that long-term strategies were not accounted for in subsequent rounds. In a real drought, the imposition of early long-term strategies would likely be of higher value. Further, having the referees chose adaptation strategies would provide a more robust platform for comparison than the votes.

\section{ACKNOWLEDGEMENTS}

The IDT was developed by Agriculture and AgriFood Canada with support from the Canada Excellence Research Chair in Water Security through the National Sciences and Engineering Research Council of Canada.

\section{REFERENCES}

Adams, J. (1995) Risk UCL Press, London.

Adams, J., and M. Thompson (2002). Taking Account of Societal Concerns about Risk: Framing the Problem. A report for the Health and Safety Executive. Available at HSE Website http://www.hse.gov.uk/research.rrpdf/rr035.pdf.

Bonsal, B. R., E.E. Wheaton, A. C., Chipanshi, C. Lin, D.J. Sauchyn, and L. Wen (2011). Drought research in Canada: a review. Atmosphere-Ocean, 49(4), 303-319.

Giacomelli P, A. Rossetti, M. Brambilla (2008). Adapting water allocation management to drought scenarios. Nat Hazards Earth Syst Sci 8:293-302

Gober, P., and H.S. Wheater (2014). Socio-hydrology and the science-policy interface: a case study of the Saskatchewan River basin. Hydrology and Earth System Sciences, 18(4), 1413-1422.

Hao, Z., J. Zheng, G. Wu, X. Zhang, and Q. Ge (2010). 1876-1878 severe drought in North China: Facts, impacts and climatic background. Chinese Science Bulletin, 55(26), 3001-3007.

Hill, H., M. Hadarits, R. Rieger, G. Strickert, E.G. Davies, and K.M. Strobbe (2014). The Invitational Drought Tournament: What is it and why is it a useful tool for drought preparedness and adaptation? Weather and Climate Extremes, 3, 107-116.

Mars, G. (Ed.). (2008). The Institutional Dynamics of Culture: The New Durkheimians (Vol. 1). Ashgate Publishing Company.

Metropolis, N., and S. Ulam (1949). The Monte Carlo Method, Journal American Statistical Association, 44(1949), pp. 335-341.

Nagourney, A. (2015). California Imposes First Mandatory Water Restrictions to Deal With Drought. The New York Times.

Nazemi, A. and H.S. Wheater (2015). On inclusion of water resource management in Earth system modelsPart 1: Problem definition and representation of water demand. Hydrology and Earth System Sciences, 19(1), 33-61. 
Strickert et al., 2015 A Stochastic Modeling Framework for the Invitational Drought Tournament

Nazemi, A., H.S. Wheater, K.P. Chun, and A. Elshorbagy (2013). A stochastic reconstruction framework for analysis of water resource system vulnerability to climate-induced changes in river flow regime. Water Resources Research, 49(1), 291-305.

Schubert, S. D., M.J. Suarez, P.J. Pegion, R.D. Koster, and J.T. Bacmeister (2004). On the cause of the 1930s Dust Bowl. Science, 303(5665), 1855-1859.

Schwarz M., and M. Thompson (1990) Divided We Stand: Redefining Politics, Technology and Social Choice. University of Pennsylvania Press.

Sivapalan, M., H.H. Savenije, and G. Blöschl (2012). Socio-hydrology: A new science of people and water. Hydrological Processes, 26(8), 1270-1276.

Strickert, G.E.H, L. Jardine, L. Bradford, (2013) Highlighting Opportunities for Stakeholder-driven Invitational Extremes Tournaments in Academic \& Non-academic Settings. A Teacher's Guide. Complied at the request of Agriculture and Agri-Food Canada - Science and Technology Branch. $31 \mathrm{pp}$.

Thompson, M. (2008a). Clumsiness: why isn't it as easy as falling of a log? Innovation: The European Journal of Social Science Research 21(3), 205-216.

Thompson, M. (2008b). Organising \& Disorganising: A Dynamic and Non-Linear Theory of Institutional Emergence and its Implications. Triarchy Press. Axminister. UK.

Thompson, M., R. Ellis, A. Wildavsky (1990). Cultural Theory: Political Cultures Series. Boulder, CO: Westview Press.

United Nations Office for Disaster Risk Reduction (UNISDR) (2009). UNISDR terminology on disaster risk reduction. Geneva, Switzerland, May

Verwiej, M., S. Luan, and M. Nowacki (2011). How to Test Cultural Theory: Suggestions for Future Research. PS: Political Science \& Politics 44(4), pp. 7450-748.

Wheater, H. and P. Gober (2013). Water security in the Canadian Prairies: science and management challenges. Philosophical Transactions of the Royal Society of London A: Mathematical, Physical and Engineering Sciences, 371(2002), 20120409.

Wheaton, E., S. Kulshreshtha, V. Wittrock, and G. Koshida (2008). Dry times: hard lessons from the Canadian drought of 2001 and 2002. The Canadian Geographer/Le Géographe canadien, 52(2), 241-262.

Wilhite, D. A. and M.H. Glantz (1985). Understanding the drought phenomenon: the role of definitions. Environmental Studies 36, 41-54. 